\title{
Survival and infection probabilities of anthropophagic anophelines from an area of high prevalence of Plasmodium falciparum in humans
}

\author{
J.D. Charlwood ${ }^{1}$, T. Smith ${ }^{2 *}$, P.F. Billingsley ${ }^{3 \dagger}$, \\ W. Takken ${ }^{4}$, E.O.K. Lyimo ${ }^{1}$ and J.H.E.T. Meuwissen ${ }^{5}$ \\ ${ }^{1}$ Ifakara Centre, PO Box 53, Ifakara, Tanzania: ${ }^{2}$ Department of Public \\ Health and Epidemiology, Swiss Tropical Institute, Socinstrasse 57, \\ Postfach, CH-4002 Basel, Switzerland: ${ }^{3}$ Department of Biology, Imperial \\ College of Science and Technology, Prince Consort Road, London SW7, \\ UK: ${ }^{4}$ Department of Entomology, Wageningen Agricultural University, \\ PO Box 8031, Wageningen, The Netherlands: ${ }^{5}$ Department of \\ Microbiology, University of Nijmegen, The Netherlands
}

\begin{abstract}
Delayed and immediate oocyst rates; parous rates and sporozoite rates were obtained in Anopheles gambiae Giles, A. arabiensis Patten and A. funestus Giles from two villages in the Kilombero Valley, southern Tanzania during the wet season of 1991. Collection methods included light trap, indoor resting collection and nets with holes cut in their side. Mosquito survival estimates from parous rates obtained from light trap collections, were compared with estimates from capture-recapture experiments and from that obtained during a population decline. Methods of estimating the proportion of feeds infectious to mosquitoes, $K$, were also compared. This proportion varied between villages and species and was highest in the village with the greatest proportion of $A$. gambiae. We propose that absolute estimates of $K$ should be obtained by determining the immediate oocyst rate and measuring the parous rate using the same host seeking mosquitoes. This estimate was only available from one village and ranged from $1.9 \%$ for $A$. gambiae s.l. to $3.4 \%$ for $A$. funestus.
\end{abstract}

\section{Introduction}

Control programmes against malaria vectors, such as the large scale use of bed nets impregnated with insecticide, limit mosquito-human contacts and hence the amount of mosquito-human transmission of malaria. They may also reduce mosquito survival. The entomological impact of such a programme can therefore be evaluated by measuring changes in the life-expectancy of the mosquitoes.

\footnotetext{
*Author for correspondence.

Present address: Department of Zoology, University of Aberdeen, Tillydrone Avenue, Aberdeen, AB9 2TN, UK.
}

Other possible interventions, in particular transmission blocking vaccines, are designed to reduce human-mosquito transmission. These might be evaluated by estimating the proportion of bloodmeals on humans which lead to malaria infections in the mosquito, (the infectious reservoir, $K$ ); or the number of potentially infectious bites arising from each bite on an infectious host (the individual vectorial capacity of the mosquito, $I$ ).

Mosquito life expectancy in the field can usually only be determined indirectly. The parous rate, $M$, defined as the proportion of adult females that have laid eggs, can be determined by dissection (Detinova, 1962) and provides estimates of survival per gonotrophic cycle. Since anophelines are gonotrophically concordant after the first 
cycle, the feeding cycle is equivalent to the oviposition cycle and $M$ can be used directly as an estimate of the survival per feeding cycle (Clements \& Paterson, 1981). Saul et al., (1990) and Burkot et al. (1990) have also shown that estimates of survival per feeding cycle can be made by comparing immediate infection (oocyst) rates with delayed rates determined in mosquitoes kept for a holding period of five days. Other methods, such as capture-recapture experiments (Service, 1993) provide estimates of survival per day.

The infectious reservoir is also difficult to quantify. Although $K$ reflects the infectious gametocyte reservoir in the human population, measurements of the proportion of people with gametocytes cannot be used to assess the number of infectious hosts. Some hosts with high density infections are not infective while some hosts with microscopically undetectable gametocytaemias are (MuirheadThompson, 1954; Graves et al., 1988; Haji et al., 1996a). Muirhead-Thompson (1954) concluded that the best way to measure $K$ was to feed uninfected mosquitoes on a random selection of hosts and to determine the delayed oocyst rate. However, in field situations, known uninfected mosquitoes that are genetically identical to local populations are usually not available and laboratory reared insects may not be as efficient transmitters. For example, estimates of $K$ based on direct feeds of laboratory reared mosquitoes from Papua New Guinea were much lower than those obtained from wild-caught anophelines (Graves et al., 1990).

One way to avoid such problems is to estimate $K$ from the increase in the proportion of mosquitoes harbouring sporozoites with increasing age (Gillies \& Wilkes, 1965; Lines et al., 1991; Charlwood et al., 1995a). However this generally requires a long series of difficult dissections. Although this method should provide a precise and unbiased measure of $K$, such estimates have been extremely variable. Lines $e t$ al. (1991) found that almost one in five feeds in Anopheles gambiae Giles (Diptera: Culicidae) on the coast of Tanzania were infectious. This was ten times higher than $K$ obtained by ourselves (Charlwood et al., 1995a) for A. arabiensis Patton in a village $200 \mathrm{~km}$ inland in the Kilombero Valley, with a similar endemicity, also in Tanzania (Smith et al., 1993; Charlwood et al., 1995a).

Another approach is to obtain $K$ from mosquito infection data alone, implicitly making use of survival estimates obtained by comparing immediate and delayed infection rates (Saul et al., 1990; Burkot et al., 1990), and avoiding the need to age mosquitoes. However, for these techniques to be applied, fed mosquitoes must be captured alive and have to survive through a holding period, a not inconsiderable practical problem.

The better a control programme works the more difficult it becomes to amass the data needed to obtain credible estimates of $K$ or $I$. Such considerations led Dye (1992) to suggest that it is more appropriate to obtain estimates of differences and changes in these quantities rather than absolute values, since if mosquito survival remains constant, the ratio $K$ (after) $/ K$ (before) intervention is approximated by the ratio of delayed oocyst rates. However, vector control measures (e.g. impregnated bed nets) should also form a part of any realistic control programme using transmission blocking vaccines, so it is unlikely that mosquito survival will remain the same. In such a programme, absolute measures of $K$ and $I$ will be useful to determine whether there is any prospect of achieving eradication, but estimates of mosquito survival will be needed even if the objective is merely to estimate the relative contributions of changes in mosquito survival and in $K$ or $I$ to transmission reduction.

The Kilombero valley is an area highly endemic for Plasmodium falciparum malaria transmitted by both $A$. gambiae s.1. (both $A$. arabiensis and $A$. gambiae s.s.) and $A$. funestus Giles (Smith et al., 1993; Charlwood et al., 1995a,b). The present study, forming part of the Kilombero Malaria Project (see Smith et al., 1993), reports parous, sporozoite and immediate oocyst rates for anophelines caught in light traps in Kilombero, and delayed oocyst rates for resting mosquitoes. We have used these data to compare several different parasitological and entomological estimates of mosquito survival and of $K$ for study villages. We advocate a simple and non-time-consuming approach which makes use of determinations of both the infection status and the parity of host-seeking mosquitoes, and which should be applicable in other areas where almost all of the malaria vectors feed on humans.

\section{Materials and methods}

\section{Study site}

The study was carried out in Namawala $\left(8^{\circ} 09^{\prime} \mathrm{S} 36^{\circ} 24^{\prime} \mathrm{E}\right.$ ) and Michenga villages $\left(8^{\circ} 07^{\prime} \mathrm{S} 36^{\circ} 39^{\prime} \mathrm{E}\right)$. The villages have been described by Charlwood et al. (1995a,b), Smith et al. (1993, 1995), and Lyimo (1993). Malaria is highly endemic in the Kilombero Valley, so that most of the village population are infected at any one time and transmission occurs throughout the year despite wide seasonal and spatial variations in vector densities (Smith et al., 1993, 1995). Population peaks of vectors occur during, and slightly after, the rains of April-May. At the start of the study, despite very high mosquito densities, less than $1 \%$ of the villagers used bednets or any other anti-mosquito devices.

\section{Mosquito sampling}

Centers for Disease Control (CDC) Light trap and 35 minute indoor resting collections were carried out as described previously (Charlwood et al., 1995a). Net-withholes collections were made using conical mosquito bednets with four $25 \mathrm{~cm}$ diameter holes cut halfway up the sides. Usual sleeping patterns were maintained. Other sleepers in the rooms were given unmodified bednets.

In Michenga village, sampling by routine light traps was carried out as described previously in about 20 houses, twice weekly, throughout the year from 1989 until early 1991 (Kilombero Malaria Project, 1992). This included houses from all sections of the village. Net-with-hole collections were made in the same houses during May 1991. Although the inhabitants had been supplied with cheap bednets two years earlier these were mostly in very poor condition and the majority of the inhabitants also slept without protection against mosquitoes. A net-with-holes was placed over each bed for one night in each of these houses. All other beds or sleeping areas were provided with new nets. Thus a house in which there were two beds was sampled for two days whereas sampling took a week to complete in a house with seven beds.

Routine light trap sampling was also carried out in Namawala as described previously (Smith et al., 1995). In addition, a light trap was run daily in a sentinel house 
(Charlwood et al., 1995b; Smith et al., 1993) in order to study temporal variation in transmission. During April-May 1991, resting collections were carried out in most of the other houses in Namawala. Houses in Namawala are mostly situated along three north-south ridges. Sampling with nets-with-holes was carried out in a series of transects along these ridges. In each of 12 houses villagers slept for four nights under nets-with-holes. Since the infectiousness of people may be age dependent (Muirhead-Thompson, 1954) a representative sample of each age group (based on census data) were included in each of these replicates.

\section{Capture-recapture experiments}

A series a capture-recapture experiments, to be described more fully elsewhere (Takken et al., unpublished), were undertaken in Namawala in the wet season of 1990. Briefly, mosquitoes caught resting inside houses were dusted with fluorescent powder in the manner described by Charlwood et al. (1986) and released inside one of the village houses. Recaptures of mosquitoes in a light trap or resting were made on the ten days following each release.

\section{Identification of mosquitoes}

Anopheles funestus and A. gambiae s.l. were distinguished morphologically. In addition, a subset of A. gambiae s.l. was stored dry over silica gel, in modified Carnoy's fixative or isopropyl alcohol and subsequently identified to species either by DNA probe using the techniques described by Hill et al. (1991) or by polymerase chain reaction (PCR) (Paskewitz \& Collins, 1990; Scott et al., 1993).

\section{Parity (M) and oocyst determination}

The proportion of host-seeking mosquitoes that had previously laid eggs (the parous rate, $M$ ) was determined by examination of the ovariolar stalks of mosquitoes collected in light traps, or by examination of tracheolar coiling in dried ovaries (Detinova, 1962). The present analyses do not distinguish between data collected using the two methods. $M$ was estimated from mosquitoes sampled over three full years.

Light trap collections were also used to determine the proportion of host seeking mosquitoes with patent oocysts (the immediate oocyst rate, $R$ ). Dissection for oocysts, on the morning after capture, consisted of cutting the terminal segment of the mosquito in $0.5 \%$ saline and gently pulling out the stomach. While stretching the stomach, oocysts were pulled to the sides rendering them easily visible. As a cross-check, all stomachs were also placed in groups of ten on slides to which a drop of $0.5 \%$ Mebromine in distilled water had been added. A cover slip was placed over the specimens, which were then examined independently for oocysts with a binocular microscope at $\times 100$ magnification.

Blood-fed and gravid mosquitoes from resting collections and blood-fed mosquitoes from net-with-holes collections were kept at ambient temperatures for five days. After this holding period the survivors were also dissected, to provide delayed oocyst rates (the proportion of these mosquitoes with oocysts) for resting mosquitoes $\left(D_{r}\right)$ and for net-withholes collections $\left(D_{n}\right)$.

\section{Sporozoite determination (S)}

Sporozoite rates $(S)$ were determined for mosquitoes collected from light traps. Dried heads and thoraxes were stored on silica gel in a freezer at $-20^{\circ} \mathrm{C}$ and later tested by ELISA (either in pools of ten or individually) for the presence of the (NANP) $)_{40}$ repeat region of the Plasmodium falciparum circumsporozoite protein (Burkot et al., 1984).

\section{Estimation of the duration of the oviposition cycle (u)}

The duration of the oviposition cycle in days, $u$, was estimated from the proportion $(A)$ of mosquitoes in light traps with dilated ovariolar stalks ('a-sacs') as described by Charlwood et al. (1985) for A. farauti. Females with sacs are assumed to have returned to feed on the day that they laid eggs (a 2 day cycle), whilst those without sacs are assumed to have delayed one day before attempting to refeed. The average duration of the oviposition cycle is then estimated as:

$$
u=3-A
$$

\section{Estimation of the duration of the extrinsic incubation period ( $E$ )}

The extrinsic incubation period $(E)$ is the number of days between an infective feed and the appearance of sporozoites in the salivary gland. A subdivision of this is $e$, the period before the appearance of visible oocysts.

$E$ is temperature dependent and for $P$. falciparum the relationship can be approximated by Moshkovsky's formula (Molineaux, 1988):

$$
E=111 /(T-16)
$$

where $T$ is the mean temperature in Celsius. Temperature data for Ifakara were used to obtain an estimate of $E$ using this formula.

Determination of the time to patency of oocysts, $e$, is problematical but under the conditions pertaining in this study, it is generally thought that oocysts become visible 4-5 days after the mosquito becomes infected.

\section{Estimation of mosquito survival}

The rate of decline in a population of $A$. arabiensis in Namawala in the absence of recruitment during FebruaryMarch 1991 provided an estimate of the daily probability of survival $(p)$. Details of how this estimate, $p_{d}$ was obtained are provided by Charlwood et al. (1995a). The decrease in daily recaptures of marked mosquitoes in the capture-recapture experiments (Takken et al., unpublished) provided a further estimate of daily survival $\left(p_{c}\right)$ (Service, 1993).

Since anophelines are gonotrophically concordant after the first cycle, the oviposition cycle is equivalent to the feeding cycle and the parous rate, $M$, can be used directly as an estimate of the survival per feeding cycle (Clements \& Paterson, 1981). This was combined with the estimate of $u$ (see above) to give an estimate of daily survival $\left(p_{M}\right)$ by assuming the latter to be approximately independent of age (i.e. an exponential survival distribution) (table 2). Similarly, the parous rate was combined with the estimate of $E$ (see above) to give an estimate, $P_{E, M}$, of the probability of surviving the extrinsic incubation period $\left(P_{E}\right)$ (table 2). Other 
estimates of $P_{E}$ were made analogously using the two other estimates of daily survival.

The proportionate increase in the infection rate associated with a single feed, is also equal to survival per feeding cycle (Saul et al., 1990). Using formulae derived from those of Burkot et al., (1990) this was used to provide estimates of survival per feeding cycle $\left(P_{r}\right.$ and $\left.P_{n}\right)$ based on infection (oocyst) rates. These estimates do not depend on either the assumption of an exponential survival distribution or on knowledge of the duration of the oviposition cycle. Similarly, equation (21) of Saul et al. (1990) using the estimated proportion infectious, $S$, and the delayed oocyst rates was also used to obtain estimates, $P_{E, r}$ and $P_{E, n}$ of survival per extrinsic cycle.

Equating estimated survival per oviposition cycle estimated from the oocyst rates to the expected value of the parous rate gives $\mathrm{E}(M)=(R / D)^{0.5}$ and $\mathrm{E}(D)=R / M^{2}$. This enabled us to make a further estimate of $P_{E},\left(P_{E M}\right)$ which made use of immediate oocyst rates and parous rates rather than delayed oocyst rates.

\section{Estimation of the infection probability of mosquitoes (K)}

Charlwood et al. (1995a) obtained an estimate, $K_{d}$, of $K$ from the rate of increase in the sporozoite rate, in the population of $A$. arabiensis in Namawala where there was negligible recruitment. In addition to this estimate, we obtained estimates of $K$ by a series of methods which used daily survival rates. At the time of capture, oocysts are evident in a proportion $R$ of the mosquitoes, consisting of: $R p$ in which oocysts remain from the previous day and $K p^{\prime \prime} / u$ mosquitoes which were infected exactly $e$ days previously and have survived to produce oocysts. We assume that the proportion of mosquitoes surviving through the entire extrinsic incubation period is small compared to those surviving long enough for oocysts to become patent, hence:

$$
R \approx R p+K p^{\prime} / u
$$

A series of estimates of $K$ termed $K_{c, d}, K_{e, c}, K_{e, M}$ can then be obtained by substituting the different estimates of $p$ in this formula (table 3 ). The analogous formula, relating the sporozoite rate $S$ to the probability of surviving the extrinsic cycle, provides a further of estimates of $K, K_{E, d}, K_{E, i}$, and $K_{e, M}$.

Saul et al. (1990) suggested the use of estimates of survival per feeding cycle rather than survival per day in determinations of the infectious reservoir and Burkot et al. (1990) provided an extension to this using immediate infection rates. They derived formulae for the infectious reservoir valid for a more general system including outdoor resting and zoophagy. Anopheles gambiae s.l. and A. funestus are both endophilic and a previous study in Kilombero found human blood indices close to 1 for both species (Charlwood et al., 1995a). We therefore used a simplified version of equation (4) of Burkot et al. (1990), to obtain estimates of $K$, termed $K_{R, r}$ and $K_{R, n}$ as functions of immediate and delayed infection rates.

Substituting the relationship between delayed and immediate rates and the parous rates into equation (4) of Burkot et al. (1990), we obtained three more estimates of $K$, $K_{R . M}, K_{M, n}$, and $K_{M . n}$ can be obtained by using $M$ and either the immediate or delayed rates. These estimates too do not require knowledge of $u$.

\section{Estimation of individual vectorial capacity (1)}

The individual vectorial capacity $(I)$ of a mosquito is the number of potentially infectious bites arising from each bite on an infectious host. This is the product of

1. The expected number of future inoculations made by a mosquito with sporozoites. Assuming exponential survival, that all feeds are on humans, and that sporozoites do not affect survival, this is $1 /\left(1-P_{1}\right)$.

2. The proportion of bites on infectious humans which result in infectious mosquitoes $=P_{E}$ (ignoring superinfections).

We used only one estimator of $I$, that based on the parous rate, $M$ :

$$
\begin{aligned}
I_{M} & =P_{E}\left(1-P_{u}\right)^{-1} \\
& =P_{E, M}(1-M)^{-1} \\
& =M^{E /(1}(1-M)^{-1}
\end{aligned}
$$

\section{Results}

\section{Mosquito collections}

In Namawala 4362 A. gambiae s.l. were collected from light traps during April and May 1991, 2177 in resting collections and 986 in net-with-hole collections. 100 of $192(52 \%)$ of these were A. gambiae and the remainder of those tested were $A$. arabiensis. The equivalent numbers for A. funestus were 4649,2349 and 1089 . The relative efficiencies of the three trapping methods were similar for the two species (test for differences in proportions; $\gamma_{2}^{2}=5.6 ; P=0.06$ ).

Mosquito densities recorded in Michenga were reported by Lyimo (1993). A greater proportion $(131 / 175=75 \%)$, of the A. gambiae s.l. identified from Michenga were A. gambiae s.s. compared with those from Namawala $\left(\gamma_{1}^{2}=20.36 ; P<0.0001\right)$. There was no difference in the proportions infected of A. gambiae s.s. or A. arabiensis in the small sample tested ( 38 A. gambiae and nine A. arabiensis uninfected versus $16 A$. gambiae and two $A$. arabiensis with oocysts; Fisher's exact test $P=0.7$ ).

Immediate oocyst rates and sporozoite rates for Namawala were determined from light trap mosquitoes collected over the whole period of 1990-1992 (Smith et al., 1993) (table 1). Immediate oocyst rates were higher in A. funestus than in A. gambiae s.l. both from the sentinel house and from other houses in the village. Delayed oocyst rates were significantly lower in the net-with-holes collection than in the resting collection for $A$. gambiae s.l. (table 1; $\left.z_{1}^{2}=11.9 ; P=0.0005\right)$ but not for A. funestus $\left(z_{1}^{2}=2.6 ; P>0.05\right)$. In $A$. gambiae s.l. the immediate oocyst rates from light trap collections were considerably lower than the delayed rates from the net with holes collection $\left(c_{1}^{2}=26.6 ; P<0.0001\right)$. In $A$. funestus this difference was small and not statistically significant $\left(\gamma_{1}^{2}=1.5 ; P=0.2\right)$. In neither species were ruptured oocysts observed. Thirty one point three percent of A. gambiae s.l. females dissected from Namawala and $61.6 \%$ of A. funestus had large ovariolar sacs (table 1) corresponding to mean oviposition cycle durations $(u)$ of 2.69 days and 2.38 days respectively.

Both delayed oocyst rates $\left(\gamma_{1}^{2}=18.8 ; P<0.0001\right)$ for May 1991 and the sporozoite rates $\left(\gamma_{1}^{2}=72.4 ; P<0.00001\right)$ for the whole period for $A$. gambiae s.l. were higher in Michenga than Namawala (table 1). This difference was much less for $A$. funestus $\left(\gamma_{1}^{2}=4.5 ; P=0.03\right)$. However, the parous rate for $A$. gambiae s.l. in Michenga was lower than that for 
Table 1. Observed characteristics of mosquitoes.

\begin{tabular}{lccccc}
\hline Quantity & \multicolumn{2}{c}{ Namawala } & \multicolumn{2}{c}{$\begin{array}{c}\text { Michenga } \\
\text { A. gambiae s.l. }\end{array}$} & A. funestus \\
\hline Parous rate & $M$ & $62.3 \%(15467)$ & $61.1 \%(9541)$ & $49 \%(2810)$ & - \\
Percentage with ovariolar sacs & $A$ & $31.3 \%(400)$ & $61.6 \%(583)$ & - & - \\
Immediate oocyst rate & $R$ & $1.9 \%(6185)$ & $3.1 \%(3859)$ & - \\
Delayed oocyst rate & & & & - \\
$\quad$ (resting catch) & $D_{r}$ & $10.4 \%(760)$ & $7.0 \%(460)$ & - & - \\
$\quad$ (net-with holes) & $D_{n}$ & $5.2 \%(578)$ & $4.3 \%(349)$ & $12.3 \%(653)$ & $5.6 \%(90)$ \\
Sporozoite prevalence & $S$ & $1.6 \%(8423)$ & $1.1 \%(10527)$ & $4.0 \%(4664)$ & $0.7 \%(3957)$ \\
\hline
\end{tabular}

Figures in brackets refer to the number of mosquitoes examined.

Namawala $\left(\chi_{1}^{2}=176 ; P<0.00001\right)$. Estimates were not available of the proportion of mosquitoes with ovariolar sacs, of the delayed oocyst rates from resting collections, or of the immediate oocyst rates from Michenga.

\section{Survival estimates}

The three estimates of daily survival of $A$. gambiae s.l. in Namawala were similar to each other (table 2), the most precise being $p_{d}$. In contrast, the estimates of survival per gonotrophic cycle varied relatively widely depending on how they were calculated. The most precise was the parous rate which was based on a very large number of dissections (table 1) and on the measurement of only a single characteristic of the mosquitoes. In A. funestus the parous rate again gave the most precise estimates, but an outlying capture-recapture experiment gave a very low estimate (0.645) of survival per day (W. Takken et al., unpublished). Standard errors are not available for the estimates derived from these capture-recapture experiments.

The average temperature recorded in Ifakara for April-May was $25.6^{\circ} \mathrm{C}$, corresponding to an estimate of the extrinsic incubation period of $E=11.6$ days. Since the precision of Moshkovsky's formula is unknown standard errors also cannot be calculated for quantities making use of this method. Most of the estimates of survival per extrinsic incubation period in Namawala were similar to each other both for A. gambiae s.l. and A. funestus, except that in both species $P_{E, n}$ was much higher than any of the other estimates (table 2). However, the anomalous capture-recapture experiment resulted in a very low estimate of survival per extrinsic incubation period $\left(P_{E . c}\right)$ and was incompatible with the observed sporozoite rate (table 2 ).

Because the parous rate for $A$. gambiae s.l. in Michenga was lower than that for Namawala, estimated daily survival and survival per cycle were also lower. The estimated survival per extrinsic incubation period $\left(P_{E, M}\right)$ based on this parous rate was also low, whilst the estimate which was calculated from the sporozoite and delayed oocyst rates $\left(P_{E, n}\right)$ was similar to that for Namawala (table 2). Because the sporozoite rate for A. funestus in Michenga was low (table 2), $P_{E, n}$ was also relatively low. This was the only estimate available for $A$. funestus in Michenga because no parity data were collected for that species.

\section{Estimates of infection probability and vectorial capacity}

Estimates of the infectious reservoirs varied from $0.7 \%$ $\left(K_{R . n}\right.$ for $A$. funestus) up to $51.3 \%$ ( $K_{E . M}$ for $A$. funestus in Michenga) (table 3). All estimates of the infectious reservoir based on estimates of daily survival for $A$. gambiae s.l. in Namawala were similar, and were similar to $K_{d}$ for A. arabiensis. The highest estimate for $A$. gambiae s.l. in Namawala, $K_{E, c}$ was $8.8 \%$. Estimates for $A$. funestus were even more variable, but some of the highest values relate to the anomalous survival estimates from the capture-recapture

Table 2. Estimates of survival rates.

\begin{tabular}{|c|c|c|c|}
\hline & $\begin{array}{c}\text { Namawala } \\
\text { A. gambiae s.l. }\end{array}$ & A. funestus & $\begin{array}{c}\text { Michenga } \\
\text { A. gambiae s.l. }\end{array}$ \\
\hline \multicolumn{4}{|c|}{ Estimates of survival per day $(p)$} \\
\hline$p_{d}$ & $0.827(0.004)$ & - & - \\
\hline$p_{c}$ & 0.813 & $0.645-0.730$ & - \\
\hline$p_{m}=M^{(1 / n)}$ & $0.839(0.025)$ & $0.813(0.013)$ & $0.77(0.018)$ \\
\hline \multicolumn{4}{|c|}{ Estimates of survival per gonotrophic cycle $\left(P_{u}\right)$} \\
\hline$M$ & $0.623(0.004)$ & $0.611(0.005)$ & $0.49(0.01)$ \\
\hline$P_{r}=\left(R / D_{r}\right)^{0-5}$ & $0.427(0.030)$ & $0.665(0.064)$ & - \\
\hline$P_{n}=\left(R / D_{n}\right)^{0.5}$ & $0.604(0.060)$ & $0.849(0.114)$ & - \\
\hline \multicolumn{4}{|c|}{ Estimates of survival per extrinsic incubation period $\left(P_{E}\right)$} \\
\hline$P_{E, d}=p_{d}^{L}$ & 0.110 & - & - \\
\hline$P_{E-r}=p_{c}^{E}$ & 0.091 & $0.006-0.026$ & - \\
\hline$P_{L M}=M^{E / u}$ & 0.130 & 0.091 & 0.046 \\
\hline$P_{E, r}=S / D_{r}$ & $0.152(0.021)$ & $0.163(0.030)$ & - \\
\hline$P_{E, n}=S / D_{n}$ & $0.304(0.061)$ & $0.367(0.069)$ & $0.324(0.041)$ \\
\hline$P_{E, R}=S M^{2} / R$ & $0.327(0.041)$ & $0.132(0.018)$ & - \\
\hline
\end{tabular}

Variance estimates were made using the formulae given in the Appendix, where appropriate. The variance of $p_{d}$ was obtained directly from the regression analysis in Charlwood et al. (1995). 
Table 3. Estimates of the mosquito infection probability.

\begin{tabular}{|c|c|c|c|c|}
\hline & $\begin{array}{l}\text { Namawala } \\
\text { A. gambiae s.l. }\end{array}$ & A. funestus (s.e.) & A. $g$ & $\begin{array}{l}\text { enga } \\
\text { s.l. (s.e.) }\end{array}$ \\
\hline$K_{d}$ & $1.8 \%(1.5)$ & - & - & \\
\hline$K_{t_{d}}=u\left(1-p_{d}\right) R p_{d}^{-t}$ & $1.9(2.3 \%$ & - & - & See note 1 \\
\hline$K_{\ell, i}=u\left(1-p_{c}\right) R p_{\mathrm{v}}^{-t}$ & $2.2(2.7 \%$ & $7.1-23.8 \%$ & - & See note 1 \\
\hline$K_{t, M}=u\left(1-p_{M}\right) R p_{M}^{-e}$ & $1.7-2.0 \%$ & $3.2-4.0 \%$ & - & See note 1 \\
\hline$K_{E, d}=u\left(1-p_{i}\right) S p_{i}^{-E}$ & $6.7 \%$ & 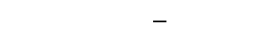 & - & \\
\hline$K_{E, c}=u\left(1-p_{c}\right) S p_{c}^{-E}$ & $8.8 \%$ & $28.2 \%$ (See note 2 ) & - & \\
\hline$K_{E, M}=u\left(1-p_{M}\right) S p_{M}^{-E}$ & $5.5 \%$ & $5.7 \%$ & $51.3 \%$ & \\
\hline$K_{R, r}=\left(\left(D_{r} R\right)^{0 . \bar{s}}-R\right) /\left(\left(R / D_{r}\right)^{0.5}-R\right)$ & $6.2 \%(0.9)$ & $2.5 \%(0.9)$ & - & \\
\hline$K_{R, n}=\left(\left(D_{n} / R\right)^{0.5}-R\right) /\left(\left(R / D_{n}\right)^{0.5}-R\right)$ & $2.1 \%(0.7)$ & $0.7 \%(0.7)$ & - & \\
\hline$K_{R, M}=(1-1 / M) /(1-M / R)$ & $1.9 \%(0.2)$ & $3.4 \%(0.3)$ & - & \\
\hline$K_{M, r}=D_{r}(M-1) /(D, M-1)$ & $4.2 \%(0.5)$ & $2.8 \%(0.3)$ & - & \\
\hline$K_{M, n}=D_{n}(M-1) /\left(D_{n} M-1\right)$ & $2.0 \%(0.5)$ & $1.7 \%(0.4)$ & $6.7 \%(0.8)$ & \\
\hline
\end{tabular}

experiments. The remaining estimates for $A$. funestus were similar to those for A. gambiae s.l. (table 3). As a consequence of the low parous rate of $A$. gambiae s.l., estimates of the infectious reservoir in Michenga were very high.

The estimate of the individual vectorial capacity $(I)$ for A. gambiae s.l. in Namawala was 0.345, and for A. funestus, 0.233 .

\section{Discussion}

\section{Estimation of survival rates}

Determinations of infection probabilities from field caught mosquitoes are highly dependent on estimates of their survival. The most unequivocal estimate of survival in Namawala was that obtained from the rate of decline of a population of $A$. arabiensis during a period without recruitment (Charlwood et al., 1995a) $\left(p_{d}\right)$. The survival rate of $A$. gambiae s.l. from Namawala determined by capture-recapture experiment was similar to $p_{t}$ as was the corresponding estimate of daily survival obtained from the parous rate $\left(p_{M}\right)$. Since parous mosquitoes can be distinguished relatively easily by dissection from nulliparous ones it is feasible to examine large numbers and hence obtain precise estimates. Parous rates therefore probably provide the most reliable indirect estimate of daily survival as long as a number of conditions are met (Charlwood et al., 1985): nulliparous and parous mosquitoes must be sampled with equal efficiency; mosquito death rate must be independent of age (which in the Kilombero appears to be the case) (Charlwood et al., 1995a); data should be collected over a whole number of complete seasons; and a good estimate of the duration of the oviposition cycle must be available.

$p_{M}$ for Michenga was rather lower than for Namawala. This might be because parous rates for Namawala cover three complete seasons whereas estimates for Michenga were only obtained from February to August 1991. If recruitment rates were lower during the missing period (which is likely because this was dry season) then survival rate estimates will be biased downwards.

Estimates of daily survival for $A$. funestus determined from parous rates in Namawala were close to those of A. gambiae s.l. Survival rates of $A$. funestus determined by capture-recapture experiment were, however, lower than those obtained by other means. This could be because marking reduces the insect's life expectancy (Burkot et al., 1990), although this does not explain why there was no bias in the estimates for A. gambiae s.l.

The limited number of mosquitoes examined for sac stages is an important contributory factor to the uncertainty in those of our estimates, such as $p_{M}$, which are a function of the duration of the oviposition cycle $(u)$. Future studies might place more emphasis on estimating this factor. However, as Saul et al . (1990) pointed out, survival per cycle, which can be estimated by $M$ directly, may be preferable to daily survival as a measure of mosquito mortality. Estimates of survival per cycle from infection data $\left(P_{r}\right.$ and $\left.P_{n}\right)$ also do not depend on $u$ and moreover do not assume an exponential survival distribution either.

Nevertheless, if mortality is age independent, $M$ is the preferred estimate of average survival per cycle because it is measured directly. The estimates of survival per cycle which used delayed oocyst rates from resting collections were on average similar to $M$, but were substantially higher when data from net-with-holes collections were used rather than resting collections from house walls. This could be the result of a bias in the sampling of the different age groups. For example, nulliparous, (hence previously uninfected) mosquitoes may have been undersampled in the conventional resting collections because they may be less likely to rest inside houses than parous ones. On the other hand, perhaps bed nets differentially deter mosquitoes of different ages from feeding, which would imply that impregnated bed nets target different sectors of the mosquito population than does spraying house walls with residual insecticide so these two strategies might complement each other. This would have operational implications for the use of bed nets as individual protection. However, we do not really know which was the most representative collection method and whilst $P_{n}$ was closest to $M$ in $A$. gambiae s.l. (suggesting that bed nets are more representative), $P$, was the closer estimate for A. funestus.

In both Namawala and Michenga delayed oocyst rates were lower in $A$. funestus than $A$, gambiae s.l. This was probably due to a reduction in the likelihood of ingesting infective gametocytes due to the smaller blood meal in the former species. Oocyst rates in A. gambiae s.l from Michenga were similar to those obtained by Babiker et al., (1995) for a hamlet of the same village and were higher than those observed in Namawala. Michenga contained the higher 
proportion of A. gambiae s.s. and this sibling species might be expected to have the highest oocyst rates because it is more strictly anthropophagic. Nevertheless, $95 \%$ of resting A. gambiae complex mosquitoes from Namawala had fed on humans (Charlwood et al., 1995b) so zoophagy in A. arabiensis from Namawala is unlikely to be the cause of the difference between villages. Estimates of survival and of $K$ made using the observed proportion of feeds on humans were very similar to those quoted, which assumed this proportion to approximate to $100 \%$.

\section{Estimation of infection probability, $K$}

The uncertainties in whether mosquitoes from walls or bed nets with holes provide representative samples of fed mosquitoes also adversely affect our confidence in those estimates of $K$ which used the delayed oocyst rates $\left(K_{E, r}, K_{E, H}\right.$ $K_{R, r}$ and $K_{R, n}$ ). This is unfortunate since these estimators have the important advantage that they did not require the estimation of any of $u, e$, or $E . K_{R, r}$ and $K_{R, n}$ are also desirable estimators in general because they do not assume exponential survival distributions for the mosquitoes.

Some of our other estimates of $K,\left(K_{e, d,}, K_{e, c}\right.$ and $\left.K_{e, M}\right)$ depended on the age at which oocysts become patent, $e$. We were unable to estimate $e$ from our data and could not therefore quantify the uncertainties associated with those estimates (which use values of $e$ taken from other studies). There is a need for more field studies estimating $e$ if these formulae are to be used.

Those estimators of $K\left(K_{t, d}, K_{E, c}\right.$ and $\left.K_{E, M}\right)$ which required the use of Moshkovsky's formula for the duration of the extrinsic cycle $(E)$ must also be viewed cautiously, since this formula must be considered an approximation and we have no estimate of its accuracy under field conditions. These estimates of $K$ for Namawala were generally much higher than the others which suggests that either the sporozoite rates or the estimate of $E$ might be biased.

$K_{R M}$ was one estimator which did not depend upon any of $E, u, e, D_{r}$ or $D_{n}$. This estimator was also the most precise among those for which we could compute confidence intervals, and also has the advantage that the two quantities needed, $M$ and $R$, can both be measured by dissection of the same host-seeking mosquitoes. Moreover, the value of $K_{R, M}$ for A. gambiae s.l. from Namawala of $1.9 \%$, was very similar to $K_{1}$, which is the most direct estimate of $K$ available for either of the study villages, and can therefore be considered as a standard against which to evaluate the other estimates. $K_{i}$ cannot, however, be recommended for general application because the approach used to estimate it (Charlwood et al., 1995a) depended on the serendipitous study of a population which was not undergoing recruitment.

$K_{t}$ was much lower than the estimate obtained using a similar approach by Lines et al. (1991) for A. gambiae s.s. in Muheza. Traditionally $A$. arabiensis is thought to be a less efficient vector than $A$. gambiae s.s due to lower survival (Gillies \& de Meillon, 1968) but this could not be the reason -for the lower rate in our study because our estimates of daily survival are very similar to those for A. gambiae in Muheza (Gillies \& Wilkes, 1965; Lines et al., 1991). Another possibility is that many A. arabiensis from Namawala had previously fed on wild animals in areas remote from human habitation (Charlwood et al., in preparation). This would not be apparent in analyses of blood meals from house resting mosquitoes but would have biased immediate oocyst rates and, to a lesser extent, delayed oocyst rates and sporozoite rates downwards, consequently affecting all of our estimates of $K$. If previously zoophagic mosquitoes are immigrating then we can expect that in the absence of control measures, there will be an increase in immediate oocyst rates (and malaria transmission) as the game animals are steadily killed for meat. Higher delayed oocyst rates than ours have been obtained from Michenga using modified delayed oocyst techniques (Babiker et al., 1995) but that work was carried out in outlying hamlets at times of declining population densities, so it is unclear whether the relatively high values were due to differences in the technique used or to the study site.

The variation in our estimates of $K$ upholds the observation of Dye (1992) that it is easier to obtain approximate estimates of changes in $K$ from mosquito infection data than to derive absolute estimates of this quantity. $K$ is, however, approximately proportional to the immediate oocyst rate, $R$, and also to the sporozoite rate, $S$. In a trial of a transmission-blocking vaccine, changes in either one or both of these quantities could be used as an approximate measure of the effect on human-mosquito transmission in the absence of measures affecting mosquito survival. Delayed oocyst rates, which are considerably more difficult to assess, offer no advantages for this purpose. However, because infection rates are generally low and change unpredictably over time (Charlwood et al., 1995a), even estimates of $R$ and $S$ are imprecise. It is also unlikely, in an integrated control programme, that mosquito survival will remain unaffected. In the absence of survival rate estimates, it would be impossible to interpret entomological assessments of programmes which also involve the use of insecticides.

$K_{R, M}$ is probably the most useful estimator of $K$ for areas of sub-Saharan Africa with anthropophagic vectors whose survival approximates the exponential distribution. We propose, as an alternative to estimates based on delayed infection rates, that absolute estimates of $K$ should be obtained by dissecting host-seeking mosquitoes to obtain both $M$ and $R$ and hence $K_{R, M}$. It is likely that evaluation of a transmission blocking vaccine will take at least a year, hence the requirement for data over a whole season is not a serious problem. Measurements of oocyst size might also be used to give improved estimates of survival and of the mosquito infection probability (Haji et al., 1996b). This strategy would be appropriate in the entomological evaluation not only of transmission blocking vaccines, but also of programmes, such as impregnated bednet schemes, that aim to kill mosquitoes or to provide personal protection.

\section{Acknowledgements}

We would like to thank the villagers of Namawala and Michenga who, as ever, cheerfully cooperated during this study. We also thank the members of the entomology group of Ifakara, especially Japhet and the other field workers in Namawala. Drs S. Hill and R. Vij kindly identified samples of A. gambiae. Richard Hayes and Penelope Vounatsou provided assistance with algebra, and two anonymous reviewers provided critical comments which enabled us to considerably improve the manuscript. Thanks to Professors M. Tanner and W. Kilama, Director General of the National Institute of Medical Research (NIMR) Tanzania for supporting the Kilombero Malaria Project of which this study was 
a part. P.F.B. was supported by the Royal Society, UK, as a University Research Fellow. Financial support was also provided by the Swiss Directorate for Technical Cooperation and Humanitarian Aid (SDC) and the Directorate General of Development Cooperation (DGIS) of the Dutch Government. Data analysis was partly supported by Swiss National Science Foundation grant 32-43527.95. Research clearance was granted by the Tanzanian Commission for Science and Technology (UTAFITI) as per ref. NSR/RCA 90.

\section{References}

Armitage, P. \& Berry, G. (1987) Statistical methods in medical research. 2nd Edn. Oxford, Blackwell.

Babiker, H.A., Charlwood, J.D., Smith, T. \& Walliker, D. (1995) Gene flow and cross-mating in Plasmodium falciparum in a Tanzanian village. Parasitology 111, 433-442.

Burkot, T.R., Zavala, F., Gwardz, R.W., Collins, F.H., Nussenzweig, R.S. \& Roberts, D.R. (1984) Identification of malaria-infected mosquitoes by a two site enzyme-linked immunosorbent assay. American Journal of Tropical Medicine and Hygiene 33, 783-788.

Burkot, T.R., Graves, P.M., Paru, R., Battistutta, D., Barnes, A. \& Saul, A. (1990) Variations in malaria transmission rates are not related to anopheline survivorship per feeding cycle. American Journal of Tropical Medicine and Hygiene 43, 321-327.

Charlwood, J.D., Birley, M.H., Dagaro, H., Paru, R. \& Holmes, P.R. (1985) Assessing survival rates of Anopheles farauti (Diptera: Culicidae) from Papua New Guinea. Journal of Animal Ecology 54, 1003-1016.

Charlwood, J.D., Graves, P.M. \& Birley, M.H. (1986) Capture-recapture studies with mosquitoes of the group of Anopheles punctulatus Donitz complex (Diptera: Culicidae) from Papua New Guinea. Bulletin of Entomological Research 76. 211-227.

Charlwood, J.D., Kihonda, J., Sama, S., Billingsley, P.F., Hadji, H., Verhave, J.P., Lyimo, E., Luttikuizen, P.C. \& Smith, T. (1995a) The rise and fall of Anopheles arabiensis (Diptera: Culicidae) in a Tanzanian village. Bulletin of Entomological Research 85, 37-44.

Charlwood, J.D., Smith, T., Kihonda, J., Heiz, B., Billingsley, P.F. \& Takken, W. (1995b) Density independent feeding success of malaria vectors (Diptera: Culicidae) in Tanzania. Bulletin of Entomological Research 85, 29-35.

Clements, A.N. \& Paterson, G.D. (1981) The analysis of mortality and survival rates in wild populations of mosquitoes. Journal of Applied Ecology 18, 373-399.

Detinova, T.S. (1962) Age-grouping methods in Diptera of medical importance, with special reference to some vectors of malaria. World Health Organization Monographs Series 47, $216 \mathrm{pp}$.

Dye, C. (1992) The analysis of parasite transmission by bloodsucking insects. Annual Review of Entomology 37, 1-19.

Gillies, M.T. \& de Meillon, B. (1968) Anophelinae of Africa south of the Sahara, (Ethiopian Zoogeographical Region). 2nd edn. Johannesburg, Publication of the South African Institute for Medical Research no, 54.

Gillies, M.T. \& Wilkes, T.J. (1965) A study of the age composition of populations of Anopheles gambiae Giles and A. funestus Giles in north-eastern Tanzania. Bulletin of Entomological Research 56, 237-262.

Graves, P.M., Burkot, T.R., Carter, R., Cattani, J.A., Lagog, M., Parker, J., Brabin, B.J., Gibson, F.D., Bradley, D.J. \&
Alpers, M.P. (1988) Measurement of malarial infectivity of human populations to mosquitoes in the Madang area, Papua New Guinea. Parasitology 96, 251-263.

Graves, P.M., Burkot, T.R., Saul, A.J., Hayes, R.J. \& Carter, R. (1990) Estimation of anopheline survival rate, vectorial capacity and mosquito infection probability from malaria infection rates in villages near Madang, Papua New Guinea. Journal of Applied Ecology 27, 134-147.

Haji, H., Smith, T., Charlwood, J.D. \& Meuwissen, J.T. (1996a) Absence of relationships between selected host factors and natural infectivity of Plasmodium falciparum in an area of high transmission. Parasitology 113, 425-431.

Haji, H., Smith, T., Meuwissen, J.T., Sauerwein, R. \& Charlwood, J.D. (1996b) Estimation of the infectious reservoir for Plasmodium falciparum in natural vector populations using oocyst size. Transactions of the Royal Society of Tropical Medicine and Hygiene 90, 494-497.

Hill, S.M., Urwin, R., Knapp, T.F. \& Crampton, J.M. (1991) Synthetic DNA probes for the identification of sibling species in the Anopheles gambiae complex. Medical and Veterinary Entomology 5, 455-463.

Kilombero Malaria Project (1992) The level of anti-sporozoite antibodies in a highly endemic malaria area and its relationship with exposure to mosquitoes. Transactions of the Royal Society of Tropical Medicine and Hygiene 86, 499-504.

Lines, J., Wilkes, T.J. \& Lyimo, E. (1991) Human malaria infectiousness measured by age-specific sporozoite rates in Anopheles gambiae in Tanzania. Parasitology 102, 167-177.

Lyimo, E.O. (1993) The bionomics of the malaria mosquito Anopheles gambiae sensu lato in Southeast Tanzania. PhD thesis, Wageningen Agricultural University.

Molineaux, L. (1988) The epidemiology of human malaria as an explanation of its distribution of its distribution, including some implications for its control. pp. 913-998 in Wernsdorfer, W.H. \& McGregor, I. (Eds) Malaria. Principles and practice of malariology. Edinburgh, Churchill Livingstone.

Muirhead-Thompson, R.C. (1954) Factors determining the true reservoir of infection of Plasmodium falciparum and Wuchereria bancrofti in a West African village. Transactions of the Royal Society of Tropical Medicine and Hygiene 48, 208-236.

Paskewitz, S.M. \& Collins, F.H. (1990) A method to distinguish mosquitoes of the Anopheles gambiae complex by the polymerase chain reaction. Medical and Veterinary Entomology 4, 367-373.

Saul, A.J., Graves, P.M. \& Kay, B.H. (1990) A cyclical model for pathogen transmission and its application to determine vectorial capacity from vector infection rates. Journal of Applied Ecology 27, 123-133.

Scott, J.A., Brogdon, W.G. \& Collins, F.H. (1993) Identification of single specimens of the Anopheles gambiae complex by the polymerase chain reaction. American Journal of Tropical Medicine and Hygiene 49, 520-529.

Service, M.W. (1993) Mosquito ecology. Field sampling methods. London and New York, Elsevier Applied Science.

Smith, T., Charlwood, J.D., Kihonda, J., Mwankusye, S., Billingsley, P.F., Meuwissen, J., Lyimo, E., Takken, W., Teuscher, T. \& Tanner, M. (1993) Absence of seasonal variation in malaria parasitaemia in an area of intense seasonal transmission. Acta Tropica 54, 55-72.

Smith, T., Charlwood, J.D., Takken, W., Tanner, M. \& Spiegelhalter, D.J. (1995) Mapping the densities of malaria vectors within a single village. Acta Tropica 59, 1-18. 


\section{Appendix}

\section{Variance formulae}

Formulae derived using the approximations given by Armitage \& Berry (1987) were used to estimate standard errors as follows:

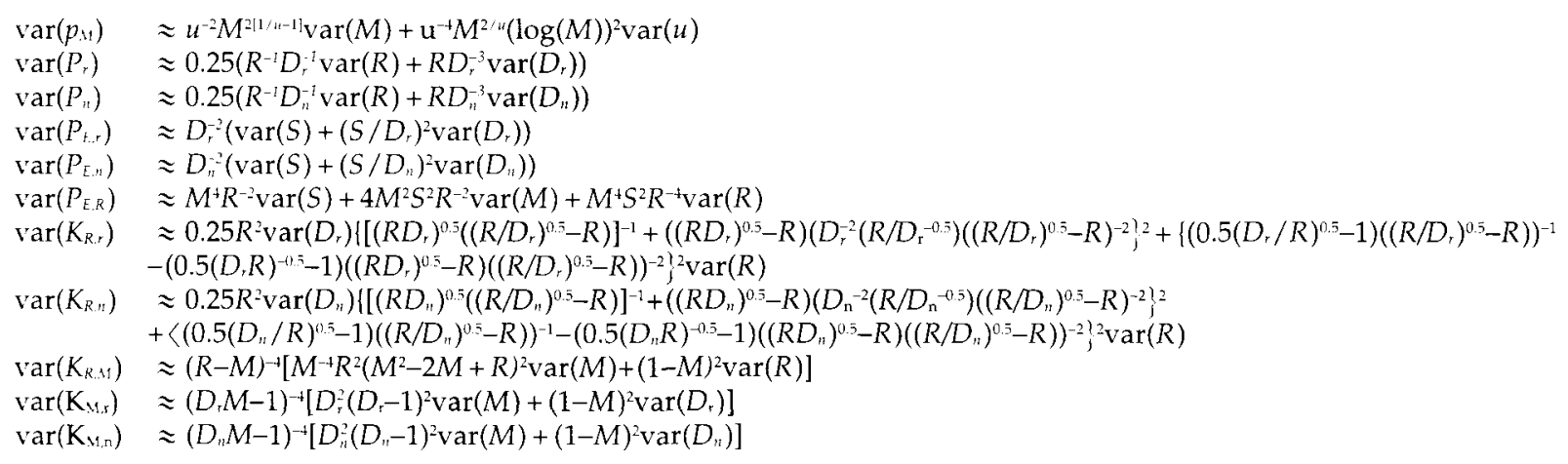

(Accepted 2 March 1997)

C. CAB INTERNATIONAL, 1997 

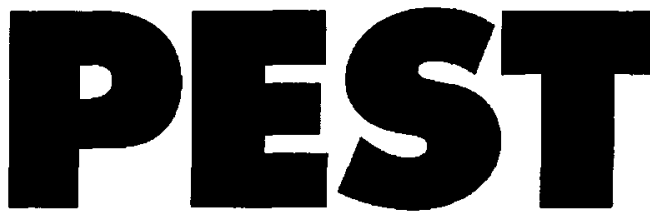

$\begin{array}{llllll}c & a & b & w & e & b\end{array}$

\section{Would you like to be able to access Bulletin of Entomological Research whenever you need to, at your own desktop?}

\section{Now you can. And there's no more to pay.}

From January 1997, CAB INTERNATIONAL launches a new World Wide Web service called CABWeb. The first phase of CABWeb, PEST CABWeb provides online access to the following pest management journals AT NO ADDITIONAL CHARGE to current subscribers:

Bulletin of Entomological Research • Review of Plant Pathology • Review of Agricultural Entomology • Nematological Abstracts • Weed Abstracts • Biocontrol News \& Information

Subscribe to any of these journals and receive the following benefits of FREE Internet access:

The information is available at the desktop or laboratory bench.

Multi-user, simultaneous access.

Standard World Wide Web technology - no need for extra training.

More timely information - no need to wait until the journal arrives in the library.

It's free! And that means no additional pressure on the library or department budget.

Each abstract journal will be enhanced with a backfile of at least 1 year's data.

\section{Find PEST CABWeb at: http://pest.cabweb.org/}

If your institution has paid for a 1997 subscription to one or more of the journals included in the service, all bona fide members of the institution then have the right to access those same titles online, at no additional cost. The user's IP (network) addresses MUST be registered before access can be granted. Ask your librarian or information officer to register on behalf of your whole institution. Individuals with personal subscriptions may register for individual access.

In order to qualify for FREE access to PEST CABWeb, the subscriber must complete, sign and return a Registration Form which can be found at the back of this journal, directly from CAB INTERNATIONAL or at http://pest.cabweb.org/

\section{CAB INTERNATIONAL}

Wallingford, OXON, OX10 8DE, UK

Tel: $+44(0) 1491832111$ Fax: +44 (0) 1491826090 E-mail: I.rogers@ cabi.org 